\title{
Presentations to general practice before a cancer diagnosis in Victoria: a cross-sectional survey
}

\author{
Karen Lacey ${ }^{1}$, James F Bishop ${ }^{1,2}$, Hannah L Cross ${ }^{1}$, Patty Chondros ${ }^{2}$, Georgios Lyratzopoulos ${ }^{3}$, Jon D Emery ${ }^{2}$
}

The known Most patients diagnosed with cancer had initially consulted a general practitioner about their symptoms. Delayed diagnosis is associated with poorer patient experience and possibly worse clinical outcomes.

The new The number of GP visits and the time from symptom onset to seeing a hospital specialist were strongly influenced by cancer type. For example, patients with pancreatic cancer or multiple myeloma were more likely to have visited a GP several times; breast cancer patients were least likely to have done so. The implications Strategies for reducing missed opportunities for diagnosing cancer earlier in general practice are needed.

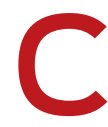

ancer is the leading cause of disease burden in Australia; by 2020 about 150000 new cases will be diagnosed each year. ${ }^{2}$ Most patients diagnosed with cancer had initially consulted a general practitioner about their symptoms; ${ }^{3-6}$ GPs are therefore pivotal in the timely diagnosis of cancer. A systematic review found evidence that longer time to diagnosis is associated with poorer clinical outcomes. ${ }^{7}$ Delays before cancer diagnosis may be caused by various factors at the patient, general practice and health system levels. ${ }^{8}$

Patients place great value in having their cancer symptoms recognised early, a priority highlighted by a number of medico-legal claims against GPs about perceived delays.,10 Multiple prediagnostic consultations with a GP are associated with a more negative patient experience of subsequent cancer-related care. ${ }^{11}$

The 2010 English Cancer Patient Experience Survey (CPES) examined GP visits preceding a cancer diagnosis, and found large variations between cancer types. ${ }^{12}$ International research has found that patient characteristics associated with primary care delays include younger age, female sex, belonging to an ethnic minority, and living in a rural area. ${ }^{5}$ However, there are few data about GP consultations and factors associated with the amount of time preceding a cancer diagnosis in Australia.

Our primary aim was to therefore assess the socio-demographic and clinical factors associated with multiple GP visits by patients subsequently diagnosed with cancer in Victoria. Our secondary aim was to investigate factors associated with the length of the interval between patients first recognising a symptom and their seeing a hospital doctor.

\section{Methods}

\section{Study design and setting}

We undertook a cross-sectional survey in 2013 of patients treated for cancer in five of the member hospitals of the Victorian Comprehensive Cancer Centre (VCCC) that cover the inner city

\section{Abstract}

Objective: To assess variations in the number of general practitioner visits preceding a cancer diagnosis, and in the length of the interval between the patient first suspecting a problem and their seeing a hospital specialist.

Design, setting and participants: Analysis of data provided to the Cancer Patient Experience Survey (CPES; survey response rate, $37.7 \%$ ) by 1552 patients with one of 19 cancer types and treated in one of five Victorian Comprehensive Cancer Centre hospitals, 1 October 2012 - 30 April 2013.

Main outcome measures: The primary outcome was the proportion of patients who had had three or more GP consultations about cancer-related health problems before being referred to hospital. The secondary outcome was the interval between the patient first suspecting a problem and their seeing a hospital specialist.

Results: $34 \%$ of the patients included in the final analyses ( 426 of 1248) had visited a GP at least three times before referral to a hospital doctor. The odds ratios (reference: rectal cancer; adjusted for age, sex, language spoken at home, and socioeconomic disadvantage index score) varied according to cancer type, being highest for pancreatic cancer (3.2; 95\% Cl, 1.02-9.9), thyroid cancer $(2.5 ; 95 \% \mathrm{Cl}, 0.9-6.6)$, vulval cancer (2.5; 95\% Cl, 0.7-8.7) and multiple myeloma (2.4; 95\% Cl, 1.1-5.5), and lowest for patients with breast cancer $(0.4 ; 95 \% \mathrm{Cl}$, $0.2-0.8)$, cervical cancer $(0.5 ; 95 \% \mathrm{Cl}, 0.1-2.1)$, endometrial cancer $(0.5 ; 95 \% \mathrm{Cl}, 0.2-1.4)$ or melanoma $(0.7 ; 95 \% \mathrm{Cl}$, 0.3-1.5). Cancer type also affected the duration of the interval from symptom onset to seeing a hospital doctor; it took at least 3 months for more than one-third of patients with prostate or colon cancer to see a hospital doctor.

Conclusion: Certain cancer types were more frequently associated with multiple GP visits, suggesting they are more challenging to recognise early. In Victoria, longer intervals from the first symptoms to seeing a hospital doctor for colon or prostate cancer may reflect poorer community symptom awareness, later GP referral, or limited access to gastroenterology and urology services.

and western suburbs of Melbourne (Peter MacCallum Cancer, Melbourne Health, Royal Women's Hospital, St Vincent's Hospital and Western Health).

\section{Data collection}

The study design was based on the English CPES. ${ }^{12,13}$ The CPES survey instrument was selected as it was cognitively validated and field-tested, and provided opportunities for international comparisons. The postal survey, provided in English, included 76 items, of which 65 assessed patient experience of diagnosis and subsequent care. Responses were closed, with most based on ordinal scales. In this article we report the results for two items about experiences prior to hospital referral (Box 1). A data 
1 Outcome questions and response options in the 2013 Victorian Comprehensive Cancer Centre (VCCC) Cancer Patient Experience Survey of patients with discharge dates, October 2012 - April 2013*

Question 1: Before you were told you needed to go to hospital about cancer, how many times did you see your GP (family doctor) about the health problem caused by cancer?

1. None - I did not see my GP before going to hospital

2. I saw my GP once

3. I saw my GP twice

4. I saw my GP 3 or 4 times

5. I saw my GP 5 or more times

6. Don't know/can't say

Question 2: How long was it from the time you first thought something might be wrong with you until you first saw a hospital

doctor?

1. Less than 3 months

2. $3-6$ months

3. 6-12 months

4. More than 12 months

5. Don't know/can't remember

* These questions are identical to those used in the National Health Service (UK) Cancer Patient Experience Survey. ${ }^{13}$

management error at one of the health services meant that patients who had declined to participate or had died could not be tracked. The expert committee therefore decided to not send reminder letters from that health service, so that only $25 \%$ of all non-responders were sent a reminder.

\section{Eligibility}

Patients were eligible to participate if they were aged 18 years or more, were discharged between 1 October 2012 and 30 April 2013 from one of the five VCCC hospitals, and had a confirmed diagnosis of cancer in the primary diagnosis field of their hospital record (International Classification of Diseases, revision 10 [ICD-10] codes C00-C99, excluding C44 [non-melanoma skin cancer]; Appendix 1). Our analysis included patients who had had at least one GP consultation about symptoms related to their subsequent cancer diagnosis. Patients were excluded if they had been diagnosed by a national screening program, had presented to a hospital emergency department, or had been admitted to hospital for an unrelated medical condition. ${ }^{12,13}$

\section{Outcomes}

The primary outcome was the proportion of patients who had had three or more GP consultations before referral to a hospital specialist for suspected cancer (Box 1, question 1). The secondary outcome was the length of the interval ( $\geq 3$ months $v<3$ months) between patients suspecting a problem until the time they first saw a hospital doctor, whether a specialist in a private facility or at a VCCC hospital (Box 1, question 2).

\section{Explanatory variables}

Analysis was restricted to the cancer types defined by ICD-10 codes used for the analysis of CPES survey data in England. ${ }^{12}$ Non-Hodgkin and Hodgkin lymphoma diagnoses were pooled, as were stomach and oesophageal cancer diagnoses, because of the small numbers of the individual cancer types in our sample.

Age was categorised as 18-34, 35-44, 45-54, 55-64, 65-74, and $\geq 75$ years. The age groups $18-24$ and $25-34$ years and $75-84$ and $\geq 85$ years were each aggregated because of the small number of participants in these categories. Sex was determined from hospital records.

Socio-economic status was assessed according to the Australian Bureau of Statistics' Index of Relative Socio-economic Disadvantage (IRSD) data for the patient's residential postcode. ${ }^{14}$

\section{Statistical analysis}

The number of GP visits prior to hospital referral about cancer and the time to hospital referral, according to the participant's age group, sex, postcode IRSD, preferred language at home, and type of cancer, were summarised as counts and percentages. Logistic regression with robust standard errors was used to estimate odds ratios (ORs) with 95\% confidence intervals for the primary and secondary outcomes with respect to each explanatory variable. Adjusted ORs (aORs) were estimated using a multivariable logistic model with robust standard errors. Sample sizes for the multivariable analyses were reduced because of missing responses for language spoken at home and postcode IRSD. Rectal cancer was used as the reference category to enable comparisons with the English CPES; it had been used as the reference in the English study because it was common in both sexes. We did not examine interactions between socio-demographic variables and cancer type because the sample size was too small. Stata 13.1 (StataCorp) was used for all analyses.

\section{Ethics approval}

Human research ethics committees at the Peter MacCallum Cancer Centre (reference, 13/11L), Melbourne Health (QA2013016), Royal Women's Hospital (approved as Quality Assurance), St Vincent's Hospital (QA 014/13) and Western Health (QA2013016) approved the study.

\section{Results}

A total of 5772 potentially eligible patients were identified from hospital records of the participating hospitals, of whom 4995 had a confirmed ICD-10 code for an eligible cancer diagnosis; 1885 of these patients $(37.7 \%)$ returned a survey, of which five with a mismatch for age, sex or diagnosis code were excluded. Of the remaining 1880 patients, 1552 had one of the 19 cancer types included in our analysis; the patients ranged in age from 20 to 95 years.

Forty-nine of the 1552 patients (3.2\%) did not respond to the question about the number of visits to a GP before referral to hospital about cancer; $1256(83.6 \%)$ stated that they had seen a GP at least once before they were referred to a hospital doctor. Five patients with testicular cancer and three patients with mesothelioma had visited a GP at least once before referral, but were excluded from further analysis because of the small numbers for these cancer types. About one-third of the remaining patients (426 of $1248 ; 34.1 \%$ ) had visited a GP at least three times before being referred to a hospital doctor.

Of the 1248 respondents who had seen a GP at least once before a hospital referral, 37 (3\%) did not respond to the question about the length of time before seeing a hospital doctor, and were therefore excluded from this particular analysis. Of the 1211 patients who responded to this question, $260(21.5 \%)$ stated that at least 3 months had elapsed between first noticing that something was wrong and seeing a hospital doctor. 


\section{Number of GP visits}

There were large variations in the proportions of patients with particular cancer types who visited their GP at least three times before referral (Box 2; Appendix 2, Figure 1). In the adjusted analysis, the odds of at least three GP visits before referral were significantly influenced by tumour type. In the adjusted analysis, patients with breast cancer $(\mathrm{aOR}, 0.4$; $95 \% \mathrm{CI}, 0.2-0.8$ ) were least likely to have had three or more GP visits before referral to hospital; patients with pancreatic cancer (aOR, 3.2; 95\% CI, 1.02-9.9) or multiple myeloma (aOR, 2.4; 95\% CI, 1.1-5.5) were most likely to have visited their GP at least three times before referral. In the unadjusted analyses, the odds of at least three GP visits before referral were significantly influenced by sex, age group, and language spoken at home, but the association with sex was lost after adjusting for age group, language spoken at home, socioeconomic disadvantage index score of patient residence, and type of cancer (Box 2).

2 Odds ratios (with 95\% confidence intervals) for patients $(n=1248)$ seeing a general practitioner about cancer-related health problems three or more times ( $v$ fewer than three times) before being advised to visit a hospital

\begin{tabular}{|c|c|c|c|c|c|c|c|c|}
\hline & Total number & $\geq 3$ GP visits & Crude odds ratio & $95 \% \mathrm{Cl}$ & $P^{*}$ & Adjusted odds ratio ${ }^{\dagger}$ & $95 \% \mathrm{Cl}$ & $P^{*}$ \\
\hline Sex & & & & & $<0.001$ & & & 0.97 \\
\hline Men & 654 & 261 (39.9\%) & 1 & & & 1 & & \\
\hline Age (years) & & & & & 0.01 & & & 0.23 \\
\hline $18-34$ & 41 & $12(29.3 \%)$ & 0.85 & $0.42-1.71$ & & 0.98 & $0.46-2.09$ & \\
\hline $55-64$ & 321 & 117 (36.4\%) & 1.17 & $0.86-1.60$ & & 1.35 & $0.96-1.88$ & \\
\hline $65-74$ & 399 & $131(32.8 \%)$ & 1 & & & 1 & & \\
\hline$\geq 75$ & 211 & $84(39.8 \%)$ & 1.35 & $0.96-1.91$ & & 1.51 & $1.03-2.22$ & \\
\hline \multicolumn{4}{|c|}{ Language spoken at home (missing responses: 51) } & & 0.01 & & & 0.08 \\
\hline
\end{tabular}

Index of Relative Socio-economic Disadvantage, by quartiles (missing responses: 7) 0.15

$\begin{array}{lcccc}4 \text { (least disadvantaged) } & 389 & 117(30.1 \%) & 1 & \\ 3 & 374 & 133(35.6 \%) & 1.28 & 0.95-1.74 \\ 2 & 294 & 102(34.7 \%) & 1.24 & 0.89-1.7 \\ 1 \text { (most disadvantaged) } & 184 & 72(39.1 \%) & 1.49 & 1.04-2.16\end{array}$

1

1.20

1.23

1.36

0.06

$1.30-12.2$

$0.94-5.98$

0.70-9.11

$1.12-5.38$

1.00-11.4

$1.10-4.22$

0.92-3.70

0.77-3.41

0.80-3.34

$0.82-4.31$

$0.70-5.40$

0.74-3.55

0.47-3.85

8 (35\%)

$15(28 \%)$

$4(25 \%)$

0.23-3.04

$29(23 \%)$

$0.36-1.52$

10 (19\%)

0.24-1.47

3 (20\%)

$0.16-2.57$

$0.22-0.90$

3.17

2.48

2.47

2.41

2.32

2.15

1.59

1.28

0.90

0.59

0.69

32 (15\%)

0.44

0.45

\subsection{6-1.66 \\ 0.87-1.73 \\ $0.90-2.06$}

$<0.001$

1.02-9.90

0.94-6.57

0.70-8.71

1.06-5.47

0.62-8.64

1.05-4.39

0.85-3.65

0.73-3.47

0.74-3.30

0.61-3.46

0.46-3.97

$0.58-2.96$

$0.42-3.88$

0.24-3.41

0.32-1.47

$0.21-1.40$

$0.10-2.06$

0.18-0.84 


\section{Time to assessment by hospital doctor}

Box 3 and Appendix 2, Figure 2 summarise the odds of an interval of at least 3 months elapsing between the patient first suspecting a problem and their first seeing a hospital doctor, according to tumour type and patient characteristics. In the unadjusted analysis, tumour type and sex were the only factors that significantly influenced the odds for this outcome. After adjustment for age, language spoken at home, socio-economic disadvantage index score of patient residence, and tumour type, the association with sex was no longer significant. Consistent with our data on multiple GP visits, intervals of 3 months or more were least likely for patients with breast cancer. In contrast, more than one-third of patients with prostate or colon cancer first saw a hospital doctor after 3 months or more.

3 Odds ratios (with 95\% confidence intervals) for an interval of at least 3 months ( $v$ less than 3 months) elapsing between patients suspecting a problem and their seeing a hospital doctor

\begin{tabular}{|c|c|c|c|c|c|c|c|c|}
\hline & Total number & $\geq 3$ months & Crude odds ratio & $95 \% \mathrm{Cl}$ & $P^{*}$ & Adjusted odds ratio ${ }^{\dagger}$ & $95 \% \mathrm{Cl}$ & $P *$ \\
\hline Sex & & & & & $<0.001$ & & & 0.19 \\
\hline Men & 635 & 165 (26.0\%) & 1 & & & 1 & & \\
\hline Women & 576 & $95(16.5 \%)$ & 0.56 & $0.42-0.75$ & & 0.77 & $0.51-1.14$ & \\
\hline Age, years & & & & & 0.83 & & & 0.76 \\
\hline $18-34$ & 41 & $10(24 \%)$ & 1.12 & $0.53-2.38$ & & 1.37 & $0.60-3.13$ & \\
\hline $35-44$ & 88 & $15(17 \%)$ & 0.71 & $0.39-1.31$ & & 1.22 & $0.62-2.40$ & \\
\hline $45-54$ & 182 & 35 (19\%) & 0.83 & $0.53-1.28$ & & 1.44 & $0.88-2.34$ & \\
\hline $55-64$ & 307 & $67(22 \%)$ & 0.97 & $0.68-1.39$ & & 1.03 & $0.70-1.52$ & \\
\hline $65-74$ & 389 & $87(22 \%)$ & 1 & & & 1 & & \\
\hline$\geq 75$ & 204 & $46(23 \%)$ & 1.01 & $0.67-1.52$ & & 1.08 & $0.71-1.66$ & \\
\hline \multicolumn{5}{|c|}{ Language spoken at home (missing responses: 48 ) } & 0.50 & & & 0.73 \\
\hline English & 1013 & $212(20.9 \%)$ & 1 & & & 1 & & \\
\hline Other & 150 & $35(23.3 \%)$ & 1.15 & $0.76-1.73$ & & 1.08 & $0.70-1.68$ & \\
\hline \multicolumn{5}{|c|}{ Index of Relative Socio-economic Disadvantage, by quartiles (missing responses: 7) } & 0.91 & & & 0.98 \\
\hline 4 (least disadvantaged) & 379 & $82(22 \%)$ & 1 & & & 1 & & \\
\hline 3 & 361 & $75(21 \%)$ & 0.95 & $0.67-1.35$ & & 0.97 & $0.67-1.42$ & \\
\hline 2 & 287 & $59(21 \%)$ & 0.94 & $0.64-1.37$ & & 1.00 & $0.67-1.48$ & \\
\hline 1 (most disadvantaged) & 177 & $41(23 \%)$ & 1.09 & $0.71-1.67$ & & 1.08 & $0.68-1.73$ & \\
\hline Type of cancer & & & & & $<0.001$ & & & 0.001 \\
\hline Colon & 46 & $16(35 \%)$ & 1.45 & $0.61-3.43$ & & 1.55 & $0.64-3.78$ & \\
\hline Prostate & 152 & $53(35 \%)$ & 1.45 & $0.72-2.92$ & & 1.52 & $0.73-3.16$ & \\
\hline Vulval & 11 & $3(27 \%)$ & 1.02 & $0.24-4.39$ & & 1.30 & $0.29-5.77$ & \\
\hline Endometrial & 52 & $13(25 \%)$ & 0.90 & $0.38-2.18$ & & 1.15 & $0.45-2.93$ & \\
\hline Lymphoma & 126 & $36(29 \%)$ & 1.09 & $0.53-2.24$ & & 1.11 & $0.52-2.39$ & \\
\hline Laryngeal & 16 & $4(25 \%)$ & 0.90 & $0.25-3.28$ & & 1.01 & $0.27-3.78$ & \\
\hline Renal/Bladder & 63 & $17(27 \%)$ & 1.00 & $0.44-2.30$ & & 1.01 & $0.42-2.38$ & \\
\hline Rectal & 52 & $14(27 \%)$ & 1 & & & 1 & & \\
\hline Cervical & 15 & $3(20 \%)$ & 0.68 & $0.17-2.77$ & & 0.93 & $0.20-4.22$ & \\
\hline Multiple myeloma & 57 & $13(23 \%)$ & 0.80 & $0.34-1.92$ & & 0.89 & $0.36-2.17$ & \\
\hline Ovarian & 22 & $4(18 \%)$ & 0.60 & $0.17-2.10$ & & 0.74 & $0.20-2.72$ & \\
\hline Melanoma & 127 & $23(18 \%)$ & 0.60 & $0.28-1.29$ & & 0.61 & $0.28-1.35$ & \\
\hline Pancreatic & 18 & $3(17 \%)$ & 0.54 & $0.14-2.17$ & & 0.59 & $0.15-2.34$ & \\
\hline Brain & 13 & $2(15 \%)$ & 0.49 & $0.10-2.51$ & & 0.53 & $0.11-2.67$ & \\
\hline Leukaemia & 76 & $12(16 \%)$ & 0.51 & $0.21-1.21$ & & 0.49 & $0.20-1.21$ & \\
\hline Oesophageal/stomach & 22 & $5(23 \%)$ & 0.80 & $0.25-2.57$ & & 0.48 & $0.12-1.91$ & \\
\hline Lung & 106 & $16(15 \%)$ & 0.48 & $0.21-1.09$ & & 0.45 & $0.19-1.07$ & \\
\hline Thyroid & 28 & $3(11 \%)$ & 0.33 & $0.08-1.25$ & & 0.35 & $0.09-1.36$ & \\
\hline Breast & 209 & $20(10 \%)$ & 0.29 & $0.13-0.62$ & & 0.33 & $0.14-0.76$ & \\
\hline
\end{tabular}

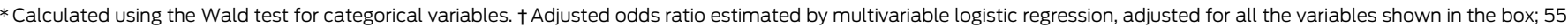
patient records were excluded because of missing responses for language spoken at home and socio-economic disadvantage index. 


\section{Discussion}

This is the first study to report Australian CPES-based data on patient experiences prior to referral to hospital about cancer. Cancer type was a strong predictor of the number of GP visits and the time from symptom onset (as judged by the patient) to first seeing a hospital specialist. Patients with myeloma or pancreatic cancer were more likely to visit a GP several times before being referred to a hospital specialist, and the interval between symptom onset and seeing a hospital specialist was more likely to be at least 3 months for people with colon or prostate cancer. Women with breast cancer were less likely to visit a GP three times before referral or to have an interval of at least 3 months between symptom onset and seeing a hospital specialist. Our findings, particularly those related to GP visits, are consistent with data from the English CPES. $^{12}$

\section{Major findings}

Our data on multiple GP visits by patients with specific cancer types suggest that certain cancers are inherently more difficult to detect in primary care, in line with earlier research and data from the English CPES. ${ }^{9,12,15}$ Pancreatic cancer and multiple myeloma present diagnostic challenges in primary care because of their nonspecific symptoms and the limited availability of easy-to-use tests for early detection. ${ }^{16,17}$ With the exception of jaundice, which is a presenting symptom in only very few cases, the positive predictive value of other common symptoms of pancreatic cancer is very low. ${ }^{18}$ In contrast, symptoms of and diagnostic tests for breast cancer are more specific, so that it is less likely that multiple GP visits occur before referral.

Compared with the data from the English CPES, ${ }^{12}$ a higher proportion of Australian patients had three or more GP consultations before they were referred to a hospital specialist (34\% v 23\%). This may reflect differences between Australian and English GPs in thresholds for referring patients suspected to have cancer, especially given the significant and sustained focus on early cancer diagnosis in England over the past decade. Alternatively, differential access to diagnostic tests may underlie this finding. Direct access to key diagnostic tests is restricted for GPs in England, whereas Australian GPs have good access to various types of investigation, particularly radiology and pathology. Investigations in primary care are associated with later referrals to a specialist, $^{19}$ as communicating the results and organising the referral may require additional consultations.

For about one-fifth of patients, more than 3 months elapsed between feeling that something was wrong and seeing a hospital doctor. This interval can be affected by several patient-, GP- and health system-related factors. ${ }^{8}$ Patients may take longer to present with certain symptoms; it may take several visits for a GP to recognise a potential cancer diagnosis and to refer the patient to a specialist; waiting times can be longer for some hospital specialists. In Australia, the mixture of public and private health care providers is complex, and we could not identify whether the period before seeing a specialist was longer for patients referred to public hospitals.

Although pancreatic and brain cancers were more likely than many cancer types to be associated with several GP consultations before referral to hospital, the overall process to seeing a specialist was less likely to take 3 months or more. This may be because of clearer and more rapid referral pathways for patients with these cancer types. The period before seeing a hospital specialist was more likely to be at least 3 months for individuals with prostate or colon cancer, possibly because patients or GPs erroneously attribute symptoms to more common, benign conditions; ${ }^{8}$ alternatively, limited access to gastroenterologists or urologists may be important. $^{20,21}$

Our data indicate that breast cancer patients were less likely to have made three or more GP visits or for 3 months to have elapsed before seeing a hospital doctor. At the patient level, this probably represents greater community awareness of breast cancer symptoms. In general practice, breast cancer symptoms are more specific and more likely to be recognised, so that patients are referred more promptly. There are also clearer diagnostic pathways for women with suspected breast cancer.

\section{Limitations}

There are limitations to this study related to the challenges of conducting large scale surveys of patients recently diagnosed with cancer. The response rate to the invitation to participate in the CPES was only $38 \%$. The VCCC had expected a response rate of $35 \%$, in line with other Australian patient experience surveys, including the Australia CANnet Consumer Survey Questionnaire $(29 \%)$ and the Victoria PROSPECT Cancer Critical Care Events survey (46\%). Response may have also been affected by the low rate $(25 \%)$ of reminder letters. The VCCC CPES was only provided to patients by mail-out (ie, no electronic version), and the survey was available only in English. Further, our sample was smaller than the sample size for the English CPES, so that the confidence intervals for our data were broader.

There was a delay of a few months between hospital discharge and the invitation to participate in the study, potentially introducing both recall and survival bias, as patients who died soon after diagnosis were not surveyed. Although we profiled the prehospital experiences of patients with 19 different cancer type diagnoses, sample limitations precluded the examination of other, rarer cancers. ${ }^{15}$

Non-response bias could not be assessed, and non-responders may have had different pre-hospital experiences, although this is of greater concern for overall mean estimates than for patterns of variation. Our study only assessed variations in patterns of care for people with a known cancer diagnosis. We do not have comparative data on referrals where a GP suspected cancer in someone who eventually received an alternative diagnosis. It is possible that some patients referred by the GP for suspected cancer may have initially visited a private facility, where their cancer was diagnosed before they underwent treatment in a VCCC hospital. Participants were asked in this survey about the length of time before first seeing a hospital doctor as a key part of their pre-diagnostic pathway and an important potential contributor to diagnostic delay. We could not identify which patients were diagnosed in a VCCC hospital or a private specialist setting, or whether different cancer diagnostic pathways affected the time between noting initial symptoms and seeing a hospital specialist. The VCCC hospitals are all metropolitan, but treat patients from across Victoria. Further investigations of the potentially complex paths of patients between public and private systems are required, and also of differences in the experiences of metropolitan and rural patients.

\section{Conclusion}

Our study provides an initial overview of the patterns of consultation prior to referral to hospital for cancer in Victoria. It shows that certain cancers are more likely to be associated 
with multiple visits to a GP before the GP refers the patient to hospital. While GPs must balance the risks of later diagnosis against overinvestigation of patients who are unlikely to have cancer, GPs may need to raise their level of suspicion for symptoms suggestive of certain cancers. ${ }^{6}$ Our data suggest that certain cancers may be more difficult to diagnose because their symptom signature is more complex. ${ }^{22}$ Earlier diagnosis of these cancers may require different approaches to those that have been successful for breast cancer. Strategies should be investigated that reduce missed opportunities for diagnosing cancer earlier in general practice, including decision support tools, fast track referral pathways, and significant event audit.

Acknowledgements: Our study was funded by the Victorian Comprehensive Cancer Centre. Georgios Lyratzopoulos is supported by a Cancer Research UK Clinician Scientist Fellowship (A18180). We thank the investigators and institutions involved in this study. We also thank all patients who responded to the surveys.

Competing interests: No relevant disclosures.

Received 20 Oct 2015, accepted 18 May 2016.

(c) 2016 AMPCo Pty Ltd. Produced with Elsevier B.V. All rights reserved.
1 Australian Institute of Health and Welfare, Australasian Association of Cancer Registries. Cancer in Australia: an overview 2012 (AlHW Cat. No. CAN 70). Canberra: AlHW, 2012. http://www.aihw.gov.au/cancer/cancer-inaustralia-overview-2012/ (accessed Oct 2015).

2 Australian Institute of Health and Welfare. Cancer incidence projections: Australia, 2011 to 2020 (AlHW Cat. No. CAN 62). Canberra: AlHW, 2012. http://www. aihw.gov.au/WorkArea/DownloadAsset.aspx?id= 10737421440 (accessed Oct 2015).

3 Hamilton W. Five misconceptions in cancer diagnosis. Br J Gen Pract 2009; 59: 441-445.

4 Rubin G, Vedsted P, Emery J. Improving cancer outcomes: better access to diagnostics in primary care could be critical. Br J Gen Pract 2011; 61: 317-318.

5 Mitchell E, Macdonald S, Campbell NC, et al. Influences on pre-hospital delay in the diagnosis of colorectal cancer: a systematic review. Br J Cancer 2008; 98: 60-70.

6 Emery JD. The challenges of early diagnosis of cancer in general practice. Med J Aust 2015; 203: 391-393. https:// www.mja.com.au/journal/2015/203/10/challengesearly-diagnosis-cancer-general-practice

7 Neal RD, Tharmanathan P, France B, et al. Is increased time to diagnosis and treatment in symptomatic cancer associated with poorer outcomes? Systematic review. Br J Cancer 2015; 112 Suppl 1: S92-S107.

8 Walter F, Webster A, Scott S, Emery J. The Andersen Model of Total Patient Delay: a systematic review of its application in cancer diagnosis. Health Serv Res Policy 2012; 17: 110-118.

9 Emery J, Chiang P. The role of risk tools in diagnosing cancer in primary care. Aust Fam Physician 2014; 43: 508-512.

10 Richards MA. The National Awareness and Early Diagnosis Initiative in England: assembling the evidence. Br J Cancer 2009; 101 Suppl 2: S1-S4.

11 Mendonca SC, Abel GA, Saunders CL, et al. Pre-referra general practitioner consultations and subsequent experience of cancer care: evidence from the English Cancer Patient Experience Survey, 2015. Eur J Cancer Care (Engl) 25: 478-490.

12 Lyratzopoulos G, Neal RD, Barbiere JM, et al. Variation in number of general practitioner consultations before hospital referral for cancer: findings from the 2010 National Cancer Patient Experience Survey in England. Lancet Oncol 2012; 13: 353-365.

13 Department of Health. National Cancer Patient Experience Survey, 2010 [computer file]. Colchester: UK Data Archive, 2010. http://dx.doi.org/10.5255/UKDASN-6742-1 (accessed Feb 2016).

14 Australian Bureau of Statistics. 2033.0.55.001. Census of Population and Housing: Socio-Economic Indexes for Areas (SEIFA), Australia, 2011. Updated Mar 2013. http:// www.abs.gov.au/ausstats/abs@.nsf/mf/2033.0.55.001/ (accessed May 2016).
15 Mendonca SC, Abel GA, Lyratzopoulos G. Pre-referral GP consultations in patients subsequently diagnosed with rarer cancers: a study of patient-reported data. $\mathrm{Br} J \mathrm{Gen}$ Pract 2016; 664: el71-el81.

16 He X-Y, Yuan Y-Z. Advances in pancreatic cancer research: moving towards early detection. World J Gastroenterol 2014; 20: 11241-11248.

17 Baur-Melnyk A, Reiser MF. Multiple myeloma. Semin Musculoskelet Radiol 2009; 13: 111-119.

18 Stapley S, Peters TJ, Neal RD, et al. The risk of pancreatic cancer in symptomatic patients in primary care: a large case-control study using electronic records. Br J Cancer 2012; 106: 1940-1944.

19 Rubin GP, Saunders CL, Abel GA, et al. Impact of investigations in general practice on timeliness of referral for patients subsequently diagnosed with cancer: analysis of national primary care audit data. Br J Cancer 2015; 112: 676-687.

20 Bobridge A, Cole S, Schoeman M, et al. The National Bowel Cancer Screening Program - consequences for practice. Aust Fam Physician 2013; 42: 141-145.

21 Emery JD, Walter FM, Gray V, et al. Diagnosing cancer in the bush: a mixed methods study of GP and specialist diagnostic intervals in rural Western Australia. Fam Pract 2013; 30: 541-550.

22 Lyratzopoulos G, Wardle J, Rubin G. Rethinking diagnostic delay in cancer: how difficult is the diagnosis? BMJ 2014; 349: g7400. 